\title{
Sensorized Tools for Haptic Force Feedback in Computer Assisted Surgery
}

\author{
Arne Sieber1,2,3, Keith Houston 1,3, Christian Woegerer3, \\ Peter Enoksson ${ }^{4}$, Arianna Menciassi ${ }^{1}$ and Paolo Dario ${ }^{1}$ \\ ${ }^{1}$ The Bio Robotics Institute, Scuola Superiore Sant'Anna, Pisa, \\ 2Imego AB, Gothenburg, \\ ${ }^{3}$ Profactor GmbH, Steyr, \\ ${ }^{4} \mathrm{MC2}$, Chalmers University of Technology, Gothenburg, \\ ${ }^{1}$ Italy \\ 2,4Sweden \\ ${ }^{3}$ Austria
}

\section{Introduction}

For centuries, open surgery was the usual way of performing an operation on a patient. Usually large incisions were required, thus this technique was traumatic for the patient and resulted in large scars and long and expensive recovery time. One paradigmatic example is cardiac surgery, where for an open surgery procedure a sternotomy is required, where the surgeon has to open with a saw the patient's sternum to access the heart and create an adequate workspace. Minimally invasive surgery (MIS) has revolutionized the way surgeries are performed in the last few decades. Here endoscopes and specialized instruments that fit in natural openings in the body or through keyhole incisions (typically 5-12 $\mathrm{mm}$ in diameter) are used and meet the patients demand for smaller incisions and shorter recovery times.

MIS techniques have numerous benefits for patients over open techniques, but unfortunately there are several drawbacks: images are usually captured from a 2D endoscopic camera and displayed on a monitor, thus the surgeon has no stereoscopic view and looses depth perception in the operating field. Another drawback is that holding rigid and long shafted instruments and controlling them at a distance leads to higher fatigue and extremely limited tactile perception. Another severe disadvantage is that mirrored motions from that of the operating field are required, as instruments are pivoting about the incision point.

\subsection{Robotic assisted minimal invasive surgery}

An alternative to traditional minimal invasive surgery is robot assisted minimal invasive surgery. Many disadvantages of manual MIS are overcome with robotics: 
- $\quad$ robot systems can emulate human hand motions without mirrored movements;

- additional degrees of freedom in the wrists are added increasing dexterity and improving instrument control;

- motion can be scaled and filtered enabling more precise motions within the patient;

- magnified 3D enhanced computer vision system provides a view of the operating site with greater clarity than open surgery (Camarillo et al., 2004).

State of the art robotic assisted surgery systems like the Da Vinci System (Intuitive Surgical, (California, USA) enable minimal invasive incisions thus offering a more conservative alternative to standard open surgery. Unfortunately, due to the lack of haptic force feedback, these systems can only be used in cases where the visual information is enough and haptic sensing is not needed (Wagner et al., 2002). Often surgeons and robotic researchers point out this fact as major limitation of robotic aided surgery (Mohr et al., 2001) (Cavasoglu et al., 2003).

A key element for enabling haptic force feedback robotic or computer assisted surgery are force sensorized tools:

"Smart medical tools" often stands for sensorized devices (Dario et al., 2003). One example are catheters with integrated pressure sensors (Strandman et al., 1997, Kalvesten et al., 1998, Melvås et al., 2002) which are mainly for diagnostic purposes, but not for surgical interventions except in the combination with so called balloon dilation for cardiac vessel expansion.

Sensorized microgrippers are described in (Houston et al., 2007). In these grippers micro strain gauges are integrated into the polymer arms, thus an accurate and precise gripping force monitoring and control is possible. On the basis of this gripper, an endoscopic tool for robotic aided surgery was developed (Houston et al., 2008) where a wire driven joint (Harada et al., 2005) allows rotation of the gripper.

Robotic aided surgical interventions where precise cutting tasks have to be performed are quite difficult - one main reason is again the lack of haptic force feedback. One approach to deal with this problem is to equip tools with Micro Electrical Mechanical Systems (MEMS) (Rebello, 2004). A force sensorized microsurgical tip is detailed in (Berkelman et al., 2003). A first prototype of a cutting blade based on a triaxial MEMS force sensor (Beccai et al., 2005) was presented in (Valdastri et al., 2005). With the help of an advanced assembly process (Sieber et al., 2007) the MEMS sensor could be directly mounted on a flexible substrate which allowed a further miniaturization of the sensorized cutting device as detailed in (Valdastri et al., 2007).

\subsection{Sensorized catheters}

Another topic addressed in this chapter is the development of sensorized tools for catheter based minimal invasive surgery. One example of a smart catheter is the recently developed TactiCath ${ }^{\circledR}$ [Endosense, Switzerland]. It is an ablation catheter, that is equipped with a force sensor to provides real time contact force measures (Vijaykumar et al., 2011).

Especially during catheter navigation through thin vessels, that can easily be damaged, haptic force feedback can improve largely the safety of such interventions. A recent approach of a "smart tool", developed especially for treatment of pulmonary atresia 
(Daubeney et al., 2005) using fetoscopy (Sydorak and Albanese, 2003), consists of a steerable and flexible catheter equipped with a fiber optic reflectance sensor for color detection, 4 electrodes for bio-impedance spectroscopy and a patented washing system (patent Sieber et al., 2007) to enable optical measurements in the presence of blood.

\subsection{Haptic force feedback input devices}

Next to force sensorized tools, haptic force feedback input devices are a key element for future computer aided surgery. One approach is to use visual force feeback. A study (Reiley et al., 2008) was carried out demonstrating that visual force feedback offers benefits like reduced suture breakage, lower forces, and decreased force inconsistencies; however it was concluded, that these benefits are mainly an advantage for novice robot-assisted surgeons and diminishing benefits among experienced surgeons.

Song et al. (2006) have developed a laboratory set up for haptic force feedback tele-operation consisting of a 6-DOF robot and a commercially available haptic force feedback input device (PHANTOM Omni). Forces on a tool (like a blade) were acquired with a 3DOF force sensor. A different group proposed to incorporate haptic force feedback into the ZEUS system by integrating a PHANTOM input device (Ortmaier et al., 2001). Another PHANTOM based system was discussed in (Tavakoli et al., 2003). In the PHANTOM haptic force feedback system, force feedback is generated with DC motors that apply a torque on the joints. Similar, but very simplified, technology is used in haptic force feedback joysticks for gaming, where the position of the joystick is acquired with 2 potentiometers and force feedback is generated by the torque of a DC motor.

For our work with the sensorized microgripper (Houston et al, 2011) we have developed a force feedback haptic tweezer. In contrast to the operating principle of the PHANTOM and haptic force feedback joysticks, the haptic tweezer is an active servo actuated tool, where the force on the tweezer is measured with a strain gauge and the tweezer is moved actively in a closed control loop. Advantages are precise movement control and low manufacturing costs. In the third part of this chapter a novel haptic force feedback joystick is described which derives from the haptic tweezer. The force on the joystick is measured with strain gauges and the joystick is actuated with servos in a control loop. The same principle can be used for designing low cost haptic input devices with 3 or more DOFs.

\section{Methods}

The methods section comprises three of our latest developments: a force sensorized cutting tool (described in Section 2.1), a steerable force sensorized catheter with haptic force feedback for fetoscopy (described in Section 2.2), and a novel joystick with haptic force feedback (described in Section 2.3) which can be used as haptic force feedback input device for the control of the sensorized cutting tool as well as the sensorized catheter.

\subsection{Force sensorized cutting tool}

Typical cutting forces in surgical interventions can reach values of up to $\pm 3 \mathrm{~N}$ for tangential and $10 \mathrm{~N}$ for normal loadings. The device described in (Valdastri et al., 2005) uses a siliconbased MEMS sensor to measure forces on a cutting blade. The MEMS sensor deployed in the 
final prototype was designed for about one tenth of these forces, thus a kinematical chain was introduced to scale down the forces on the cutting blade to the MEMS force range.

Unfortunately the viscoelastic behavior of the polyurethane kinematical chain allowed repeatable results only for alternating loadings, but not for static ones. Moreover the limited force resolution of $50 \mathrm{mN}$ was not sufficient for a precise haptic force feedback. Additionally the tool turned out to be quite fragile: Applying strong tangential forces to the blade often resulted in a detachment of the blade support and breakage of the silicon structure. While further developments of that prototype are still ongoing, in parallel to that, a complete redesign of the force sensorized cutting tool was initiated with focus on:

- $\quad$ accurate and precise force readings;

- linear force readings over a wide force range;

- $\quad$ at least 10 times higher force resolution;

- high mechanical robustness.

The main idea behind the development of the novel tool is to design a mechanical structure from a strong and tough material (stainless steel) that deflects linearly when a normal or tangential force is applied in the required force range. On the support micro strain gauges are bonded, which allows the direct measurement of the elastic deflections of the structure thus simplifying the whole device. Thus a kinematical chain as in (Valdastri et al., 2005) can be avoided (the viscoelastic behavior of the kinematical chain was the main reason for inaccurate readings for static forces).

Figure 1a details the first design of the cutting tool. Mechanical simulations were carried out in Comsol Multyphysics (figure 1a) to calculate optimum dimensions, whilst keeping the overall size to a minimum and mechanical performance to a maximum. The most important criteria are that the sensor can function to a maximum load of $\pm 3 \mathrm{~N}$ tangential and $\pm 20 \mathrm{~N}$ normal and that the force readings are linear and have low hysteresis. The first structure prototype parts were fabricated using micro-Wire-EDM from a plate of $400 \mu \mathrm{m}$ thick stainless steel.

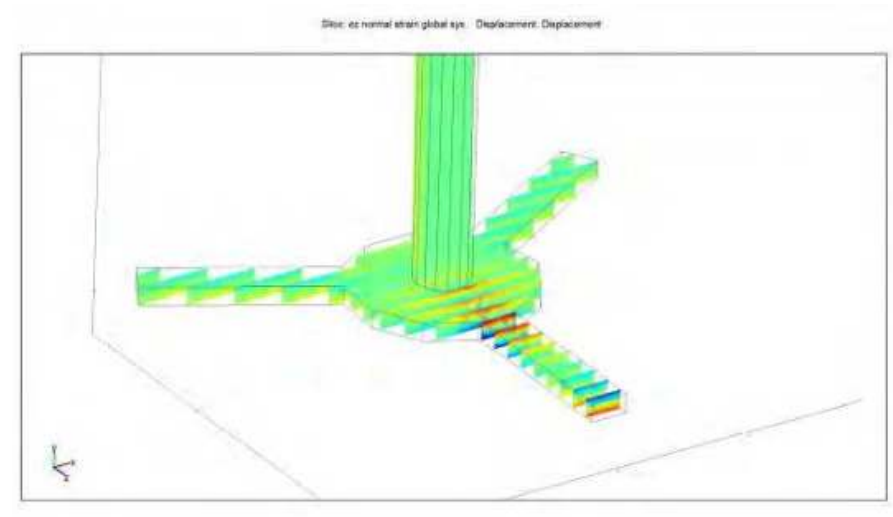

a

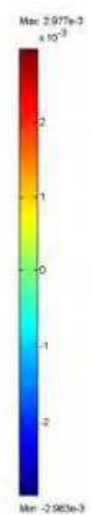

Fig. 1. a. FEMLAB simulation; b. Tool tip design

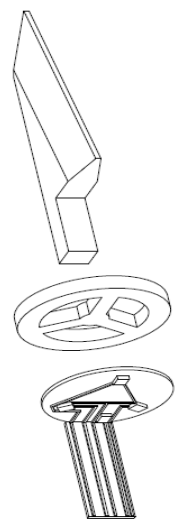

b 
After these parts were cut out and prepared the cutting blade is brazed to the stainless steel structure using a special holding fixture. The cutting blade used was a conventional scalpel blade cut with wire EDM to smaller dimensions, both to reduce the size of the overall tool and also to allow a proper fit to the other parts of the sensor. To measure the deflection of the 3 support arms, micro fabricated strain gauges from Vishay on a flexible substrate were then bonded to the structure with M-600 epoxy from Vishay. For assembly of the first prototype separate micro strain gauges were used to simplify the construction. These strain gauges were of an overall area of approximately $2 \mathrm{~mm} \times 2 \mathrm{~mm}$ (contact pads included, foil grid of $380 \mu \mathrm{m} \times 500 \mu \mathrm{m}$ ) and excess material was cut out to leave a strain gauge of approximately $450 \mu \mathrm{m} \times 1000 \mu \mathrm{m}$. In reality mass production requires an automatized industrial assembly process but for the first prototype the manual steps are sufficient. In the final design the three micro strain gauges are incorporated with the necessary connections on a common flexible substrate, which would make assembly much easier (figure 1b).

Once the strain gauges were mounted (Fig 2a), the next step was to bond wires to the strain gauges by soldering and then set the wires so that they can be easily taken out of the tool for connection to a suitable connector. To house the sensor an aluminum tube of diameter $4 \mathrm{~mm}$ was chosen. Recesses were machined into the top part of the tube to allow mounting of the sensor tip. The sensor tip is then carefully placed into the tube with all wires and then set into a fixture. The final step is to form the top lip of the aluminum tube over the sensor, thus partially closing the sensor and mechanically clamping it to the tube. For the prototype a manual fixture was used to close the structure but for a more automated approach with higher tolerances and overall quality a customized press would be necessary. A Polyoxymethylene (POM) support was developed for a first characterisation of the device (Fig 2b).

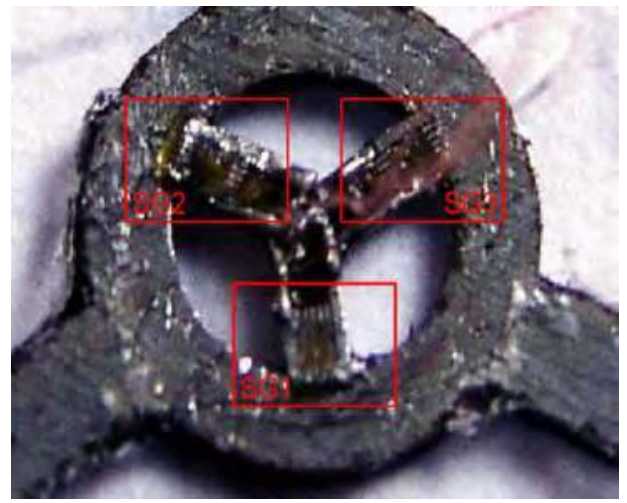

a

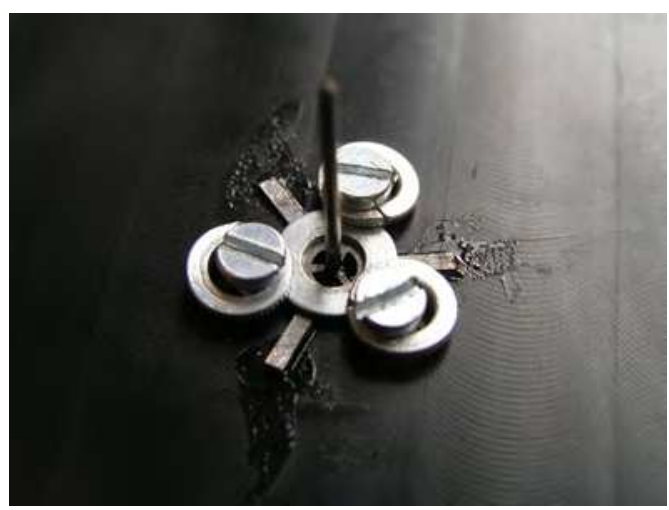

b

Fig. 2. a. Micro strain gauges mounted on the steel structure; b. Demonstrator ready for characterisation

Figure 3 details the processing of the strain gauges (Rsg1, Rsg2 and Rsg3) in $1 / 4$ Wheatstone bridge configuration. A reference voltage of $2.5 \mathrm{~V}$ is generated with the two resistors Rref1 and Rref 2 with $10 \mathrm{k} \Omega$ each. Three potentiometers are integrated for offset removal. 
For processing of the three analog channels a 24bit sigma delta AD converters [AD7730, Analog Devices] are used, which are with their programmable input range from $10 \mathrm{mV}$ to +$80 \mathrm{mV}$ especially designed for sensor signal processing. Reference voltages as low as $1 \mathrm{~V}$ are possible. Moreover a programmable digital filter can be used to suppress noise.

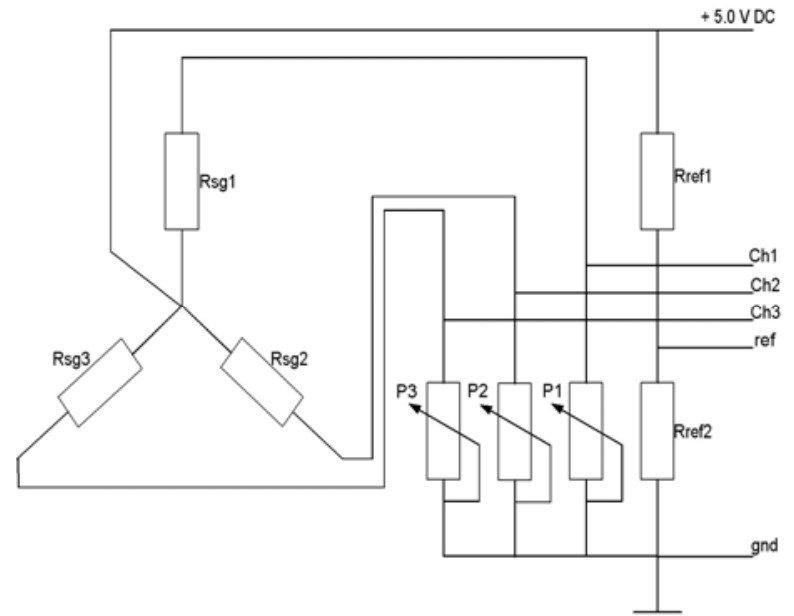

Fig. 3. Strain gauges in $1 / 4$ Wheatstone Bridge configuration

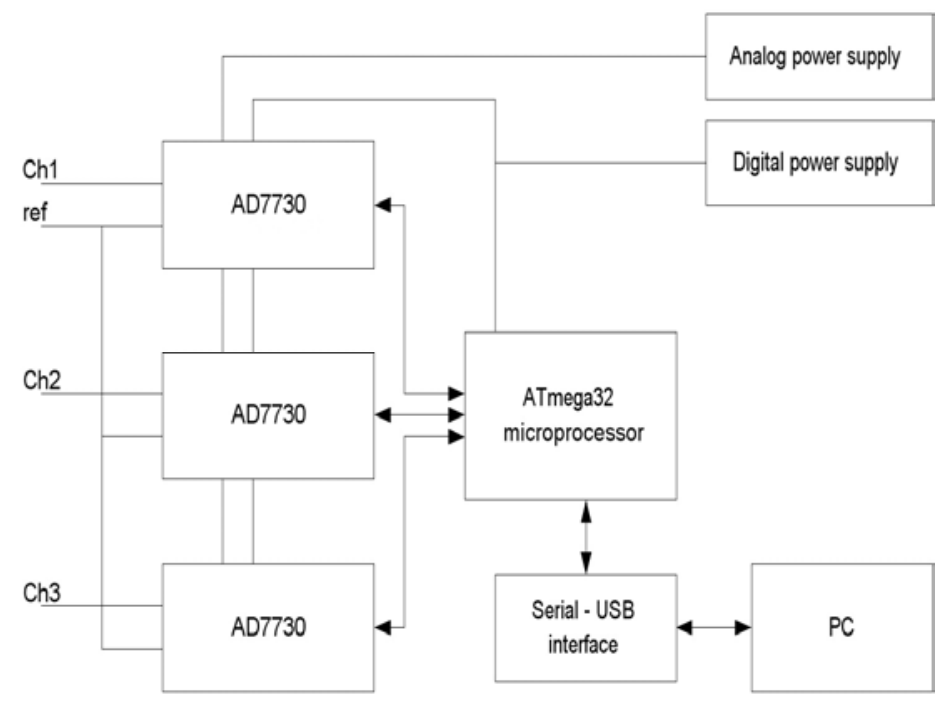

Fig. 4. Interfacing the AD converters

In a first prototype of the electronics the $\mathrm{AD}$ converters were directly connected to the parallel port of a PC and then interfaced with software developed in LabView 8.5 [National Instruments]. The software emulated serial peripheral interface did not perform flawlessly, thus in the final version (Fig 4) the three analog AD converters are interfaced to a 8 bit RISC 
microcontroller [Atmel, ATmega32] and then connected to the PC via a serial to USB interface [FT232, Future Technology Devices International Limited, United Kingdom]. The firmware for the microcontroller was developed in AVR Studio [Atmel] and the WinAVR GNU C Compiler. Measurement data are then processed and visualized with a GUI under LabView.

Several prototypes with various lengths (figure $5 \mathrm{a}, \mathrm{b}$ and $6 \mathrm{a}, \mathrm{b}$ ) were manufactured and characterized. In the following the main technical data of the prototypes are listed:

Length: 16mm (including support):

Diameter of the device: $4 \mathrm{~mm}$

Tangential forces: $\max \pm 3 \mathrm{~N}, 4 \mathrm{mN}$ resolution

Normal forces: $\max 20 \mathrm{~N}, 50 \mathrm{mN}$ resolution

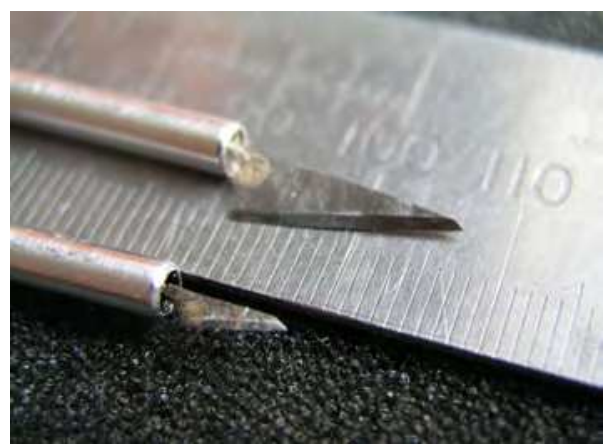

a

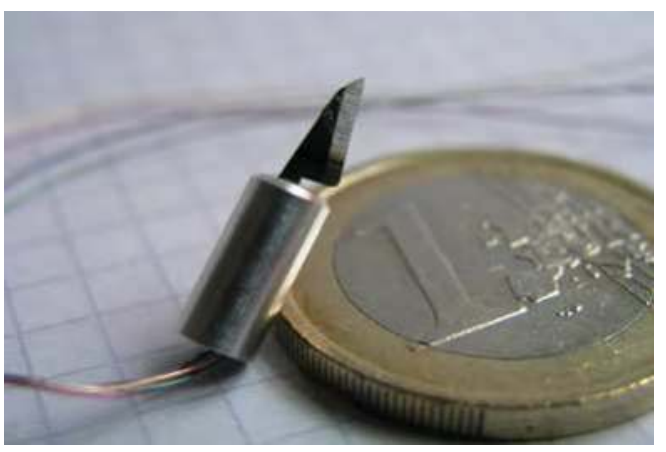

b

Fig. 5. a. Prototypes with an overall length of 25 and $50 \mathrm{~mm}$ including the support structure; b. Prototype of total length $20 \mathrm{~mm}$

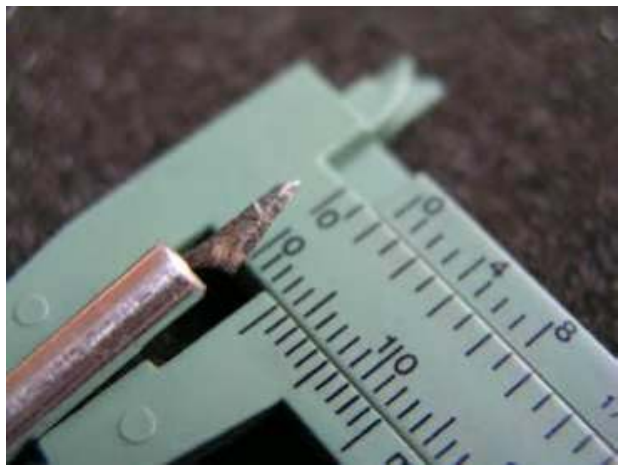

a

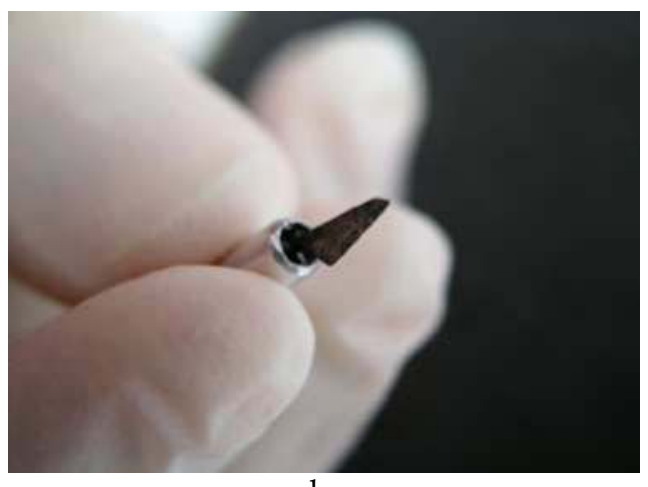

$\mathrm{b}$

Fig. 6. Final prototype has an outer diameter of $4 \mathrm{~mm}$

In a first characterisation of the device the output signals were compared to the ones of a commercial available load cell in a setup similar to (Valdastri et al., 2007). The sensor gives linear readings of strain gauge output versus load. The sensor is also robust which is necessary for surgical applications. Its small size (4mm diameter) makes it suitable for robotic aided surgery. 


\subsection{Sensorized catheter}

\subsubsection{Introduction to fetoscopy}

For a recently aborted fetus with diagnosed congenital malformation, continuation of the pregnancy until termination with a Cesarean delivery, change in timing mode, or place of delivery are the only three options available. Fetal surgery may provide a further solution in these cases. Open fetal surgery is state of the art (Harrison, 2003) (Berris and Shoham, 2006) and is already available in over a dozen clinical centers worldwide. Endoscopic fetal surgery, which is commonly referred as Fetoscopy (Sydorak and Albanese, 2003) is however a medical discipline that is still in its early development stages. Fetal surgery has given the possibility to treat some fetal congenital malformation in utero (diaphragmatic hernia, myelomeningocele, lower urinary tract obstruction).

The R\&D of a surgical tool for interventions on the fetus during pregnancy requires highly advanced micro/nanotechnologies from the system integration point of view and a close collaboration with medical doctors to develop it according to their real needs. At present fetal surgery lacks purposely developed micro instrumentations because paediatric surgeons are trying to apply standard minimal invasive instruments to fetal surgery applications. These instruments are far too large for interventions like treatment of pulmonary atresia in such early stage and also do not offer capabilities such as tissue classification.

\subsubsection{Pulmonary atresia}

During pregnancy the oxygen necessary is not supplied through the fetal lungs but by the placenta. The Foramen Ovale is an opening between the right and the left atrium, that allows blood to pass by the ventricles. After birth this opening is usually closed. Pulmonary Atresia is a malfunction that may appear during pregnancy: it is an incorrectly developed pulmonary valve. Instead of a valve there is just a membrane. Thus no blood supply to the lungs is possible, and this causes the death of newborns when oxygen supplied by the placenta is not supplied anymore. Pulmonary atresia occurs in about one out of every 20,000 live births. (Daubeney et al., 2005) (Litovsky et al., 2005)

Anatomic obstruction to the right or left ventricular outflow tract may cause ventricular dysfunction, can divert fetal blood flow in the uterus and result in cardiac chamber hyperplasia. Thus severe aortic or pulmonary stenosis can result in a hypoplastic left or right ventricle with an inability for the ventricular chambers to support the systemic or pulmonary circulation. Theoretically early relief of the fetal aortic or pulmonary stenosis may prevent such occurrence and might preserve the right or left ventricular function .

Pulmonary atresia can be diagnosed in the 12-14th week of gestation. The surgical intervention should be performed as soon as possible. In the 14th week the fetus size is about $9-14 \mathrm{~cm}$ and has a weight in the range of $60-200 \mathrm{~g}$. In this development stage the pulmonary membrane has a diameter of approximately $1 \mathrm{~mm}$.

In the case of pulmonary atresia an early surgical intervention is the only alternative to abortion and could allow normal development of the pulmonary valve and the right ventricle. A suitable surgical procedure for the treatment of pulmonary atresia is by accessing the right ventricle with a steerable micro catheter, identifying the pulmonary valve/membrane and then perforating it. However one must consider that tissues in this 
early development state are very fragile and can be damaged easily, thus haptic force feedback is a important safety feature in order to avoid to damage to the vessels or the heart when navigation the catheter, which would be life threatening for the fetus.

\subsubsection{Catheter design}

The catheter prototype is fabricated from polyurethane with a flexible end of $40 \mathrm{~mm}$ length and an outer diameter of $3,5 \mathrm{~mm}$. The diameter of the main inner lumen is $2,5 \mathrm{~mm}$. Four 380 $\mu \mathrm{m}$ thin lumen are integrated in the wall (thickness: $550 \mu \mathrm{m}$ ) and serve as guidance for four Bowden cables. A micromachined tip (Fig 7a and 7b) fabricated from Polyether Ether Ketone (PEEK) is bonded to the flexible end of the catheter and mainly acts as a support for four impedance measurement electrodes and two optical fibers. Furthermore it offers a main channel leading to the inner lumen of the catheter. This inner lumen serves as a supply of washing solution (physiological saline solution).

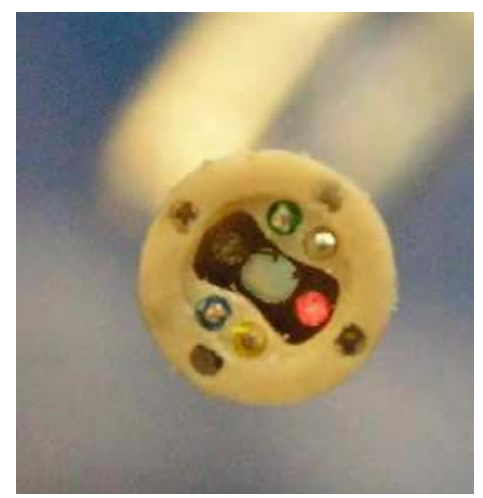

a

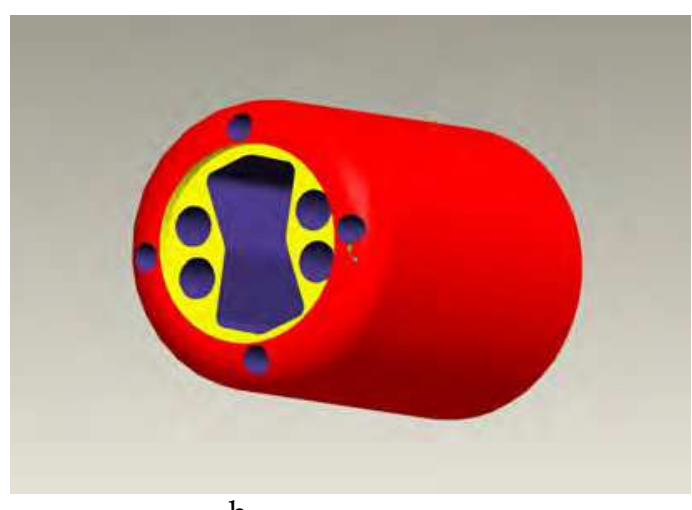

b

Fig. 7. a. Catheter tip prototype; b. Catheter tip 3D model

To be able to reach the point of interest, the catheter needs to be equipped with steering capabilities. The multi lumen catheter consists of a very flexible ending and a less flexible part. In the walls of the catheter four lumen are integrated, each one for one steering wire. Such wires are usually referred as Bowden cables. Pulling on these four wires and releasing at the same time the wire which is on the opposite side in the catheter will primarily result in a bending movement of the flexible end part of the catheter. Two microcontroller driven servo drives are used to pull and release the wires. This microcontroller is then connected to a personal computer, which is equipped with a haptic force feedback joystick allowing a precise control of the catheter. A third degree of freedom is realized by either manually or servo supported driving the catheter forward and backwards. The arm on the servo controller, which is actuating the Bowden wire is equipped with a strain gauge, which allows measurement of the actuation forces on the Bowden wire. As the actuation force reflects the force required to bend the catheter tip, it also reflects a force on the catheter tip itself, thus it can be used for haptic force feedback. For the readout of the strain gauges a similar circuit, but just with two AD converters, like described in 2.1, figure 4 was used. Figure 8 shows the first prototype of the catheter controlled by a commercial joystick. 
For electrical characterisation of tissues in front of the sensors an electrical impedance sensor was integrated. Bio-impedance spectroscopy allows tissue classification and identification by recording and analyzing the electrical impedance at different frequencies. From the electrical point of view cell membranes behave like capacitors. In comparison to low frequency electrical current where the current path is leading mainly through extra cellular fluid, high frequency electrical current is able to penetrate the cells. Thus impedance over frequency and phase over frequency plots (or both combined in "Wessel plots") are characteristics of tissues as they reflect the electrical characteristics of the cells and the tissue composition. Principle Component Analysis can then be used to classify a tissue by a recorded data set. For impedance spectroscopy two or four electrodes configuration are state of the art. Four electrodes impedance measurement allows higher accuracy, as two electrodes are used to drive in the electrical current and the other two, which are normally arranged in between the first two ones, are used for measuring the voltage drop, so called four probe measurements. The electrical contact impedance (usually current dependent) of the two voltage sensing electrodes can when be neglected, as the sensing input of impedance meter is high - so there is almost no current flowing.

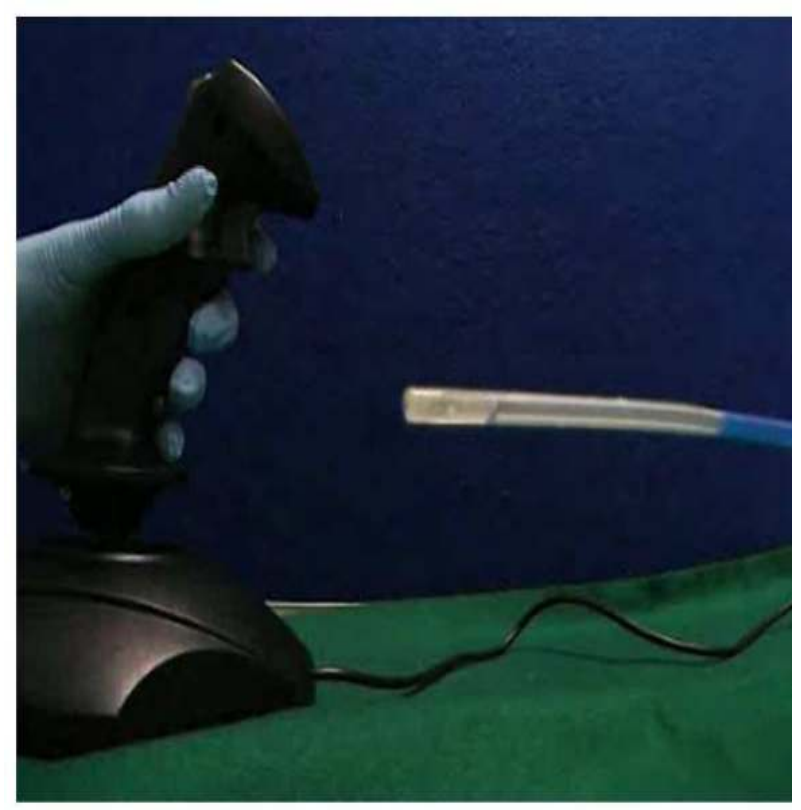

Fig. 8. Operation of the steerable catheter tip

In comparison to the four electrodes measurement technique the two electrodes configuration has the disadvantage of sensing the voltage through the same electrodes which are used to drive the electrical current. The recordings therefore show tissue impedance in serial with the electrode impedances. A two electrode configuration has the advantage of needing less space, which is an important aspect in designing a miniaturized catheter. Furthermore the two electrodes configuration can be divided into a small electrode on the tip of the catheter and a large area counter electrode which is attached to the body. 
The recorded impedance spectrum is then mainly defined by the electrical impedance characteristics of the tissue exactly in front of the small sensing electrode. This electrode configuration is especially interesting from the miniaturisation point of view, as the centre electrode could then also be used for radio frequency cutting needed for the perforation of the pulmonary membrane.

It must be kept in mind that for tissue classification it is not necessary to record accurate impedance data from the electrical point of view. It is important that the training data sets are recorded with the same electrode configuration to give comparable recordings.

Even if a two electrode configuration may be suitable for tissue classification, four electrodes are integrated in the peek tip of this first catheter prototype so that two and four electrodes configurations can be compared to each other. In the test setup a TEGAM 3550 LCR Meter is used to record impedance data. It is connected to a Personal Computer through a GPIB link.

Another way of characterisation of tissues is optical spectroscopy. Therefore two optical fibers were integrated in the front of the catheters tip. A suitable light source illuminates the tissue with light guided to the point of interest through an optical fiber. Reflected light is then received with a second fiber leading to an optical spectrophotometer. The spectral range must be chosen according to the ranges where the tissues have characteristic reflectance spectra.

In normal conditions the heart is filled with blood. Haemoglobin is a strong light absorber, where the wavelength dependent light absorption is furthermore dependent on the oxygenation status of haemoglobin. Measuring tissue characteristics in such an environment with a spectrophotometric method is not possible. The solution for this problem was solved with the integration of another lumen in the catheter to provide washing solution. With a small amount of physiological saline solution blood in front of the catheters tip can be washed away. Blood in the measuring zone is thus substituted with the optical clear washing solution, which enables spectrophotometric reflectance measurement.

Impedance spectrum recording and spectrophotometric spectrum recording requires steady state conditions - the tissue in front of the catheter should not move relatively to the catheter, which is difficult to realize in a beating heart environment. To solve this problem the washing system describes above can be used in a second way: After the blood is washed away with a small amount of physiological washing solution, the washing solution pump will be used to suck in washing solution thus creating suction in front of the catheter which will suck the tissue in front of the catheter to the tip thus establishing a reliable electrical connection to the electrode(s) and steady conditions for the measurements. A differential pressure sensor is integrated to allow precise suction force control.

Once tissue is classified as "pulmonary valve tissue" the perforation of the membrane is the next step. In this case an electrode is used for RF cutting. This electrode can either be a separate electrode or one of the impedance electrodes on the catheters tip. For this task a tissue fixation as described above is also a great advantage, as it allows a safe and controlled perforation in a quasi-static environment. Figure 9 shows the principle function of the catheter for electrical and optical tissue characterisation 


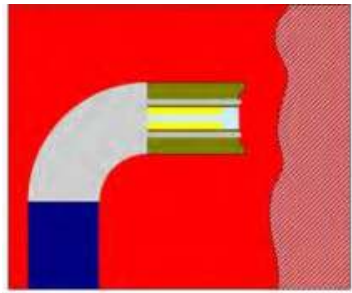

1) approaching the tissue

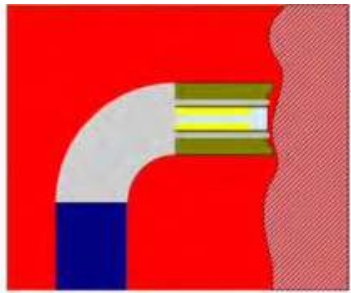

2) touching

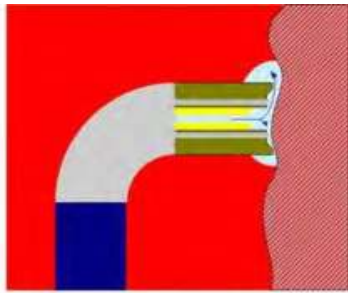

3) washing

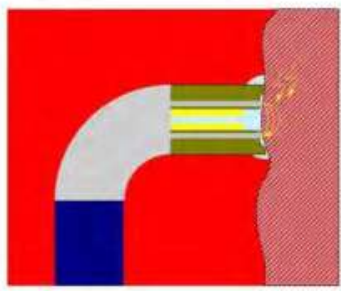

Applying suction: 4) Impedance spectroscopy

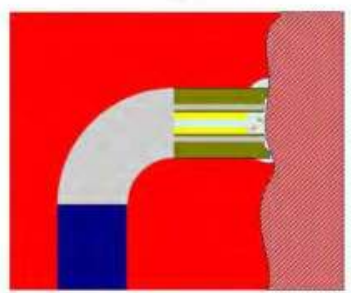

5) optical spectroscopy

Fig. 9. Multifunctional catheter tip for impedance and optical spectroscopy with integrated suction fixature

\subsection{Research and development of a haptic force feedback joystick}

In the first instance, the steerable catheter described in 2.2. was actuated with a commercial available low cost force feedback joystick. This kind of joysticks is typically used for playing computer games like flight simulators to achieve a more realistic gaming experience. The position of the joystick is sensed with potentiometers and haptic force feedback is generated by DC motors. An interface was developed under National Instruments Labview to read in the joystick coordinates and to apply forces with the DC motors. First tests revealed that the precision of the force feedback motors is rather insufficient and cannot be precisely controlled and the maximum torque is rather low. A precise force feedback comparable to operator experiences when using an expensive haptic input device like the Phantom is not possible with this kind of joystick.

For the control of the catheter a device is necessary that allows:

- $\quad$ precise control of the force feedback

- force feedback torques up to $1 \mathrm{Nm}$

- fast control to allow smooth force feedback to the operator

- $\quad$ smooth movement without binding

- " "intelligent" control of feedback to give the right "feel"

\subsubsection{Sensor / actuator design approach}

Previously we have developed a haptic tweezer for force feedback microgripping. Different from devices like the PHANTOM of the previously tested commercial force feedback joysticks, where the position of the inpout device is sensed and then a torque/force is applied with a motor, forces on the microgripper are sensed with strain gauges and the 
tweezer arms are actively moved with a microservo. The closed loop control does not only allow a precise control of the forces excerted by the haptic tweezer to the fingers, but also the behavior of the device can be programmed. In the easiest form, the "virtual" spring constants of the tweezer can be set.

The main idea for the R\&D of our force feedback joystick was to use the same sensing/actuation principle we used for the haptic tweezer for a device with more DOFs. A first 1DOF demonstrator was built consisting of a high torque servo and a $12 \mathrm{~cm}$ PEEK handle mounted on the servo actuator. The diameter of the handle was $12 \mathrm{~mm}$, but reduced to a square profile of $5 \times 5 \mathrm{~mm}^{2}$ in a $15 \mathrm{~mm}$ long section close to the axis of rotation of the handle in the high strain area. Here 2 strain gauges were positioned in this area.

A microcontroller (Atmel ATmega32L) was used to read out the strain gauges and control the servo. A closed loop control was implemented allowing to

- $\quad$ read in the signal on the strain gauges

- calculate the actuation torque

- compare the actuation torque with the position and the force feedback model (like for example a spring model)

- $\quad$ set the servo position

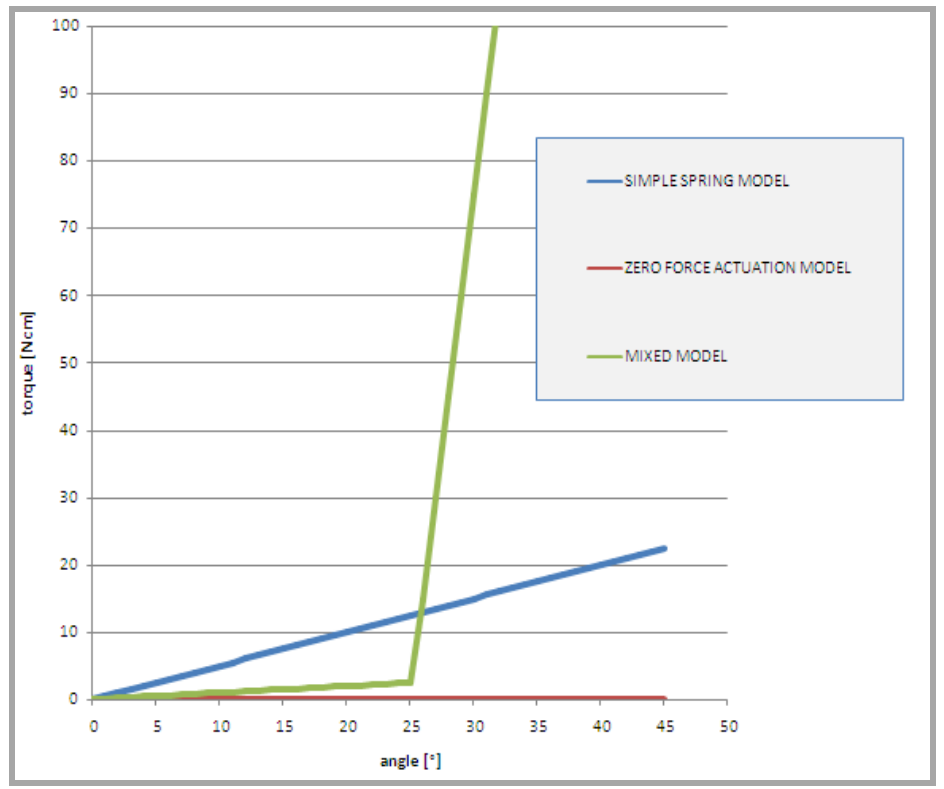

Fig. 10. Torque on the handle of the actuator

Simple scenarios were successfully tested, including the simple spring model (the torque on the handle increases with the angle), zero force actuation (a minimum force is required to move the handle) and mixtures of both. The latter was especially important as it reflects the requirements for the catheter control. When the catheter tip is freely moving without touching, zero actuation force is used. Alternatively, one might consider to program a low 
spring constant to move the joystick back to central position when released. When at a certain angle the catheter touches for instance a wall, a force is sensed in the bowden wires. This force signal is then used to generate a force feedback signal. Figure 10 shows the torques applied by the servo motor in these 3 scenarios. In the simple spring model the torque is directly dependent on the angle $\left(0.5 \mathrm{~N} \mathrm{~cm} /{ }^{\circ}\right)$. In the zero actuation force model practically no force is required to move the handle. If the handle is released, it stays at the last position. In the mixed model, a low spring constant was programmed for $0-25^{\circ}$, above that a spring constant of $10 \mathrm{Ncm} /{ }^{\circ}$ was implemented to simulate touching. If the handle is released, it returns to the central position.

\subsubsection{Design of the force feedback joystick}

Fig.11 shows a CAD plot of the original prototype design. Principal design objectives were that the device should be low cost therefore plastics were extensively used, only the actual control stick (\#5) is made of (metal) aluminium. The other parts shown in Fig.11 (support brackets \#2,3,6, base plate \#3) are made of POM. Parts 1 \& 6 are digital servo motors (GRAUPNER DS8311) and were specifically chosen for their durability (metal bearings), precision (13 bit), compactness and high torque output (112 Ncm @ 6V supply) values.

To enable force sensing and closed loop force feedback 4 strain gauges are placed at the base of the control stick (Part \#8) which will allow the force felt by the user in the $X$ \& Y axes to be measured. Screws and bearings as well as the electronic control board (integrated into the base plate) are not shown. The base plate has dimensions $140 \mathrm{~mm} \times 140 \mathrm{~mm}$.

The idea of the haptic force feedback joystick was now to add another pair of strain gauges and add a second servo actuator for force feedback on the second axis.

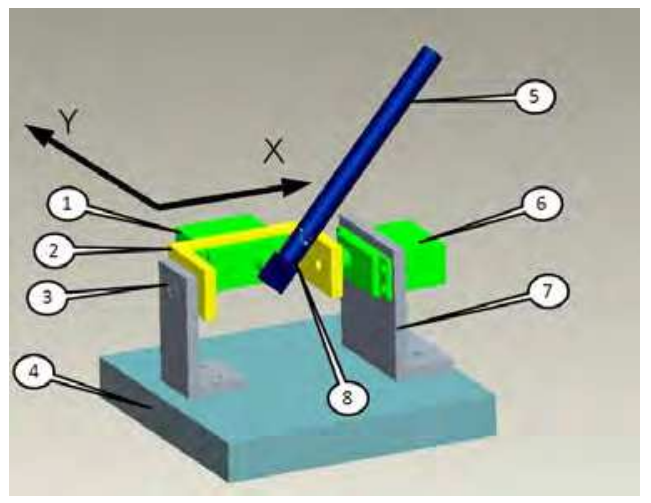

Fig. 11. CAD design of prototype joystick

Fig. 12a shows the fabricated prototype being tested by a user. All plastic parts were milled from stock POM material while the control stick was turned and then the surfaces for the strain gauges were milled flat. The strain gauges are protected from damage by a rigid plastic cover (\#2). Note the servo control cables leading from the servos to the control board under the base plate (\#1). Fig. 12b shows a larger view of the sensorized control stick (plastic cover removed) with the 4 strain gauges (\#1-4) and the electrical connector (\#5). 


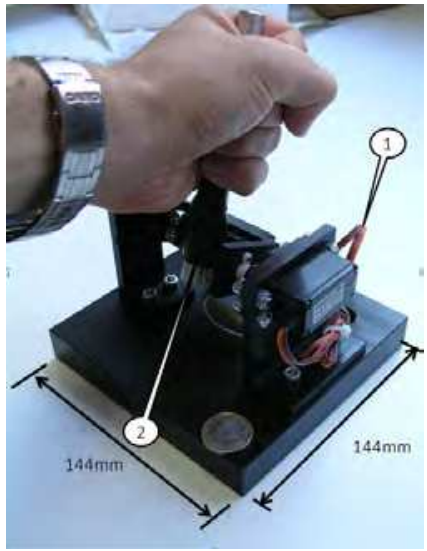

a

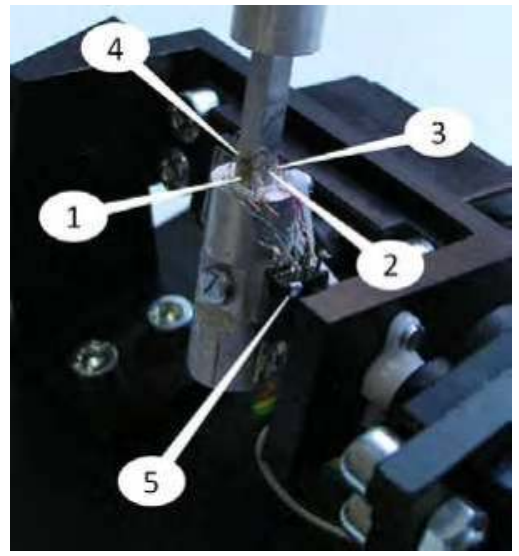

b

Fig. 12. a. Fabricated joystick prototype; b. Strain gauges and connector on control stick

Fig.13a shows the electronic control board which is fixed in a cavity under the base plate. This cavity was milled out of the bottom of the base plate. The control system purpose is to control the servo motors to give a resistive force to the user which is determined by the hand movements of the user and the control algorithms used by the microcontroller to vary the user experience. The strain gauges are in a Wheatstone bridge configuration and the differential voltages are fed to the voltage amplifiers ( $2 \times$ AD623, \#3). The amplified voltages (which represent the resistive force felt by the user) are processed by the ATMEL ATmega32 microcontroller (\#2) which through a specific algorithm then send digital commands to the servo motors (\#4) to continually adjust this force. The force sensed by the user and the position of the control stick in both $\mathrm{X} \& \mathrm{Y}$ axes can be communicated to a PC by the microcontroller. The microcontroller transmits the data to the RS232 chip (\#1) which communicates to the external device connected to \#6 with the RS232 protocol. The board is powered with a regulated $6 \mathrm{~V}$ supply connected at \#5.
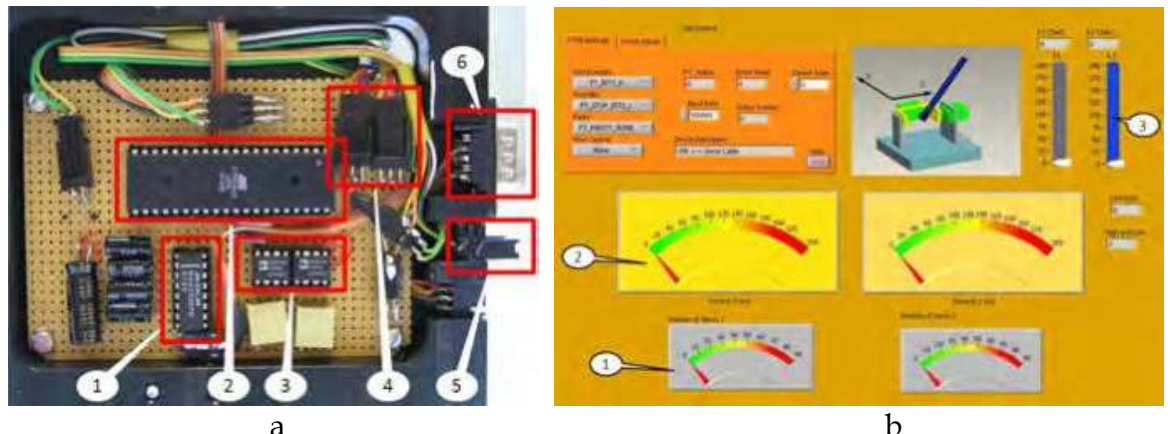

b

Fig. 13. a. Electronic control board; b. Graphical User Interface

Fig.13b shows a PC user interface developed using Labview which was used to test the performance of the input device in registering both control stick position (\#1) and force (\#2) and also using the PC interface to change the stiffness (resistance) of the control stick sensed 
by the user. Only simple trials were performed with linear spring constants to vary the stiffness of the control stick but for future trials more advanced algorithms will be programmed to also feedback non-linear and damped forces to the user.

A second prototype was designed with the aim of further reducing the size of the input device. Fig.14 shows the CAD design of the second prototype which has a base plate dimension reduced from $140 \times 140 \mathrm{~mm}^{2}$ to $84 \times 84 \mathrm{~mm}^{2}$ (40\% reduction). The same servos will be used in a different configuration and the supporting brackets have been modified to make more efficient use of space. The material for all load bearing parts (base plate, brackets, control stick) will be aluminium to make the design more robust but will also slightly increase the manufacturing costs.

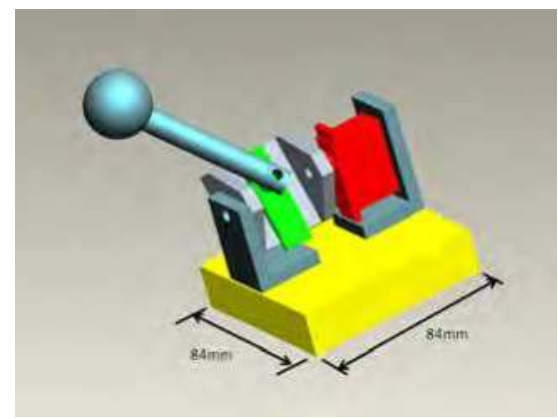

Fig. 14. CAD model of second prototype with $40 \%$ size reduction

\section{Conclusion}

The sensorized surgical blade, with its large force range and high resolution, is perfectly suitable for minimal invasive surgery and has the potential to lead to a new generation of endoscopic tools for robotic aided surgery capable also of providing precise and accurate haptic force feedback. The tool can withstand a normal load of $20 \mathrm{~N}$ and a tangential load of $3 \mathrm{~N}$ without damage to the structure. At the same time, it is possible to perform sensitive touching and probing movements with high force sensitivity down to the $\mathrm{mN}$ range.

In applications where the crucial point of the surgical intervention is not accessible with rigid manipulators, a steerable catheter can be helpful. A novel device was developed that is equipped with a steerable tip, but also with an electrical and an optical sensor system for tissue characterisation. The catheter tip is steered by a joystick. First insertion and navigation tests in phantoms were successfully carried out. The current catheter diameter is $3.5 \mathrm{~mm}$, but for applications in severe cases like pulmonary atresia, a further miniaturisation to an outer diameter of $0.8 \mathrm{~mm}$ is necessary and still feasible. Forces on the bowden wires are detected with strain gauges and used to generate haptic force feedback.

Commercial available force feedback joysticks turned out not to be precise enough for haptic sensing, thus a prototype device was developed to satisfy these additional requirements. Differently from existing force feedback input devices, where the position is sensed and then a torque is applied by a motor, in this device the force is first sensed and then the position of the joystick is set according to the programmed force/angle model within a closed loop control. A design for a second prototype joystick with reduced dimensions and improved 
electronics was also presented. Based on the same force sensing/control approach a further development of an haptic input device with 3 or more DOF is possible, which can be a low cost alternative to devices like the Phantom.

Force sensorized tool and haptic force feedback input devices for surgical interventions are still in early development stages and only few devices are already used in surgical interventions, but latest developments are promising. These novel devices have the potential to revolutionize robotic aided surgery, as the can be considered as key elements to regain the sensation of feeling tissues in a robot dominated surgical scenario.

\section{Acknowledgment}

The work described in this paper was supported by the ASSEMIC project, Marie Curie Research \& Training Network (MRTN-CT- 2003-504826). Currently Arne Sieber is funded through the Marie Curie Project Lifeloop (EU FP7-People-IEF-2008, project nr. 237128). A special mention to Mr. C. Filippeschi for his continuous and invaluable help.

\section{References}

Beccai, L., Roccella, S., Arena, A., Valvo, F., Valdastri, P., Menciassi, A., Carrozza, M.C., Dario, P. (2005) Design and fabrication of a hybrid silicon three-axial force sensor for biomechanical applications. Sensors and Actuators A, 120, 2005, pp. 370-382.

Berkelman, P.J., Whitcomb, L.L., Taylor, R.H., Jensen, P. (2003) A miniature microsurgical instrument tip force sensor for enhanced force feedback during robotassisted manipulation, 2003, pp. 917-922.

Berris, M., Shoham, M. (2006). Febotics - a marriage of fetal surgery and robotics, Computer Aided Surgery, July 2006; 11(4): 175-180

Camarillo, D.B., Krummel, T.M., Salisbury, L.K. (2004). Robotic technology in surgery: past, present, and future. Am. J. Surg., 188(4A):2S-15S, 2004.

Cavusoglu, M.C., Williams, W., Tendick, F., Sastry, S.S. (2003) Robotics for telesurgery: Second generation berkeley/ucsf laparoscopic telesurgical workstation and looking towards the future applications. Ind. Rob., 30(1):22-29, 2003.

Dario, P., Hannaford, B., Menciassi, A. (2003). Smart surgical tools and augmenting devices. IEEE Trans. Robot. Automat., 19(5), 2003., pp. 782-792.

Daubeney, P.E.F., Wang, D., Delany, D.J., Keeton, B.R., Anderson, R.H., Slavik, Z., Flather, M., Webber, S.A. (2005), Pulmonary atresia with intact ventricular septum: predictors of early and medium-term outcome in a population-based study. J Thorac Cardiovasc Surg 2005; 130:1071.

Harada, K., Tsubouchi, K., Fujie, M.G., Chiba, T. (2005). Micro Manipulators for Intrauterine Fetal Surgery in an Open MRI, Proceedings of the 2005 IEEE International Conference on Robotics and Automation Barcelona, Spain, 2005

Harrison, M.R. (2003). Fetal Surgery: Trials, Tribulations, and Turf, Journal of Pediatrics Surgery, 2003, Vol. 38, pp. 275-282.

Houston, K., Sieber, A., Eder, C., Menciassi, A., Dario, P. (2008). Novel Haptic Tool and Input Device for Bilateral Biomanipulation addressing Endoscopic Surgery, Biodevices 2008, Funchal, Portugal, 2008

Houston, K., Sieber, A., Eder, C., Tonet, O., Menciassi, A., Dario, P. (2007). Novel Haptic Tool and Input Device for Real Time Bilateral Biomanipulation addressing Endoscopic Surgery, EMBC 2007, Lyon, France, 2007 
Houston, K., Sieber, A., Eder, C., Vittorio, O., Menciassi, A., Darion, P., (2011), A Teleoperation System with Novel Haptic Device for Micro-Manipulation, International Journal of Robotics \& Automation, Vol. 26, No. 3, 2011

Kalvesten, E., Smith, L., Tenerz, L., Stemme, G.,. The first surface micromachined pressure sensor for cardiovascular pressure measurements, MEMS 98. Proceedings., The Eleventh Annual International Workshop on Micro Electro Mechanical Systems, 1998, Page(s): 574 - 579

Litovsky, S., Choy, M., Park, J., Parrish, M., Waters, B., Nagashima, M., Praagh, R.V., Praag, S.V. (2000), Absent pulmonary valve with tricuspid atresia or severe tricuspid stenosis: report of three cases and review of the literature., Pediatr Dev Pathol 3(4), 353--366.

Melvås, P., Kälvesten, E., Enoksson, P.,Stemme, G. A free-hanging strain-gauge for ultraminiaturized pressure sensors, Sensors and Actuators A: Physical, Volumes 97-98, 1 April 2002, Pages 75-82

Mohr, F.W., Falk, V., Diegeler, A., Walther, T., Gummert, J.F., Bucerius, J., Jacobs, S., Autschbach, R. (2001). Computer-enhanced "robotic" cardiac surgery: Experience in 148 patients. J. Thor. Cardio. Surg., 121(5):842-853, 2001.

Ortmaier, T., Reintsema, D., Seibold, U., Hagn, U., Hirzinger, G. (2001) The DLR minimally invasive robotivs surgery scenario. Workshop on Advances in Interactive Multimodal Telepresence Systems, Munich, Germany, March 2001

Rebello K. (2004). Applications of MEMS in surgery. Proc. IEEE, 92(1), 2004., pp. 43-55

Reiley, C.E., Akinbiyi, T., Burschka, D., Chang, D.C., Okamura, A.M., Yuh, D.D. (2008) Effects of visual force feedback on robot-assisted surgical task performance. Thorac Cardiovasc Surg 2008;135:196-202

Sieber, A., Valdastri, P., Houston, K., Menciassi, A., Dario, P. (2007) Flip Chip Microassembly of a Triaxial Force Sensor on Flexible Substrates, Journal of Sensors and Actuators, 2007

Song, G., Guo, S., Wang, Q. (2006), A Tele-operation system based on haptic feedback. Proceedings of the 2006 IEEE International Conference on Information Acquisition August 20 - 23, 2006, Weihai, Shandong, China

Strandman, C., Smith, L., Tenerez, L., Hoek, B. (1997). A production process of silicon sensor elements for a fibre- optic pressure sensor. Sensors and Actuators A, 63(1), 1997. pp. 69-74

Sydorak, R.M., Albanese, C.T. (2003). Minimal access techniques for fetal surgery, World J Surg, 2003, Vol. 27, pp. 95-102.

Tavakoli, M., Patel, R.V., Moallem, M. (2003) A Force Reflective Master- Slave System for Minimally Invasive Surgery. Proceedings of the 2003 IEEE/RSJ Intl. Conference on Intelligent Robots and Systems, Las Vegas, Nevada, 2003.

Valdastri, P., Sieber, A., Houston, K., Menciassi, A., Dario, P., Ynangihara, M., Fujie, M. (2007) Miniaturized Cutting Tool With Triaxial Force Sensing Capabilities for Minimally Invasive Surgery. ASME Journal of Medical Devices, Sept. 2007

Valdastri, P., Harada, K., Menciassi, A., Beccai, L., Stefanini, C., Fujie, M., Dario, P. (2005) Integration of a miniaturised three axial force sensor in a minimally invasive surgical tool, IEEE Trans. Biomed. Eng, 2005

Vijaykumar, R., Locke, A.H., Kralovec, S., Neuzil, P., Fonck, E., Harari, D., Reddy, VZ.(2011) Spatiotemporal Distribution of Catheter-Tissue Contact Force across the Left Atrium during Pulmonary Vein solation, Heart Rhythm Society, San Fransisco, CA, PO6-85, May 2011

Wagner C.R., Stylopoulos, N., Howe, R.D. (2002). Proc. of the tenth symposium on haptic interfaces for virtual environment and teleopertator systems, 2002, pp73-79 


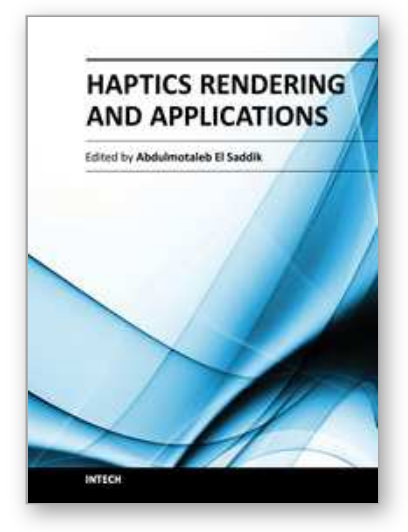

\author{
Haptics Rendering and Applications \\ Edited by Dr. Abdulmotaleb El Saddik
}

ISBN 978-953-307-897-7

Hard cover, 246 pages

Publisher InTech

Published online 27, January, 2012

Published in print edition January, 2012

There has been significant progress in haptic technologies but the incorporation of haptics into virtual environments is still in its infancy. A wide range of the new society's human activities including communication, education, art, entertainment, commerce and science would forever change if we learned how to capture, manipulate and reproduce haptic sensory stimuli that are nearly indistinguishable from reality. For the field to move forward, many commercial and technological barriers need to be overcome. By rendering how objects feel through haptic technology, we communicate information that might reflect a desire to speak a physicallybased language that has never been explored before. Due to constant improvement in haptics technology and increasing levels of research into and development of haptics-related algorithms, protocols and devices, there is a belief that haptics technology has a promising future.

\title{
How to reference
}

In order to correctly reference this scholarly work, feel free to copy and paste the following:

Arne Sieber, Keith Houston, Christian Woegerer, Peter Enoksson, Arianna Menciassi and Paolo Dario (2012). Sensorized Tools for Haptic Force Feedback in Computer Assisted Surgery, Haptics Rendering and Applications, Dr. Abdulmotaleb El Saddik (Ed.), ISBN: 978-953-307-897-7, InTech, Available from: http://www.intechopen.com/books/haptics-rendering-and-applications/sensorized-tools-for-haptic-forcefeedback-in-computer-assisted-surgery

\section{INTECH}

open science | open minds

\author{
InTech Europe \\ University Campus STeP Ri \\ Slavka Krautzeka 83/A \\ 51000 Rijeka, Croatia \\ Phone: +385 (51) 770447 \\ Fax: +385 (51) 686166 \\ www.intechopen.com
}

\author{
InTech China \\ Unit 405, Office Block, Hotel Equatorial Shanghai \\ No.65, Yan An Road (West), Shanghai, 200040, China \\ 中国上海市延安西路65号上海国际贵都大饭店办公楼 405 单元 \\ Phone: +86-21-62489820 \\ Fax: $+86-21-62489821$
}


(C) 2012 The Author(s). Licensee IntechOpen. This is an open access article distributed under the terms of the Creative Commons Attribution 3.0 License, which permits unrestricted use, distribution, and reproduction in any medium, provided the original work is properly cited. 